\title{
Assessment of Image Quality Parameters for Computed Tomography in the City of Yaoundé
}

\section{Calvin Didier Njiki, Joseph Eric Martial Ndjaka Manyol, Yolande Ebele Yigbedeck, Daniel William Abou'ou, Blaise Clovis Yimele, Jean Faustin Sabouang}

National Radiation Protection Agency, Yaoundé, Cameroon

Email:cdnjiki@yahoo.fr

How to cite this paper: Njiki, C.D., Ndjaka Manyol, J.E.M., Ebele Yigbedeck, Y., Abou'ou, D.W., Yimele, B.C. and Sabouang, J.F. (2018) Assessment of Image Quality Parameters for Computed Tomography in the City of Yaoundé. Open Journal of Radiology, 8, 37-44.

https://doi.org/10.4236/ojrad.2018.81005

Received: February 5, 2018

Accepted: March 10, 2018

Published: March 13, 2018

Copyright $\odot 2018$ by authors and Scientific Research Publishing Inc. This work is licensed under the Creative Commons Attribution International License (CC BY 4.0).

http://creativecommons.org/licenses/by/4.0/

\begin{abstract}
Performance evaluation of computed tomography is not significant in the Republic of Cameroon in general and more particularly in the city of Yaoundé. Therefore, this work aimed to analyze image performances of $05 \mathrm{CT}$ scanners in 05 medical facilities in the city of Yaoundé, by using the Catphan 700 phantom and test procedures of the Atomic Energy Agency. Five quality control tests were performed on each CT-Scanner. All the CT scanners evaluated showed good results and were not disapproved in three of the five tests performed. The difference between the measured spatial resolution for each of the five CT scanners and the spatial resolution specified by the manufacturer was very high. The low contrast resolution of four of the five CT scanners was found low when compared to the low contrast resolution specified by the manufacturers.
\end{abstract}

\section{Keywords}

CT, Image Quality, Catphan 700

\section{Introduction}

Quality assurance (QA) methods in diagnostic radiology aims to promote the effective use of radiation through achieving and maintaining appropriate image quality while delivering a minimal, dose to the patient.

Routine quality control (QC) procedures in computed tomography (CT) centers in Cameroon are not given much attention due to no/limited certified staffs trained to carry out this task.

The IAEA recommends acceptance tests and periodically QA-tests of CT-scanners with respect to radiation dose and image quality [1]. Some important parameters are radiation profile width, CT-numbers, uniformity, spatial 
resolution and low contrast resolution. Low contrast resolution is the ability to differentiate objects with slightly different densities. The potential to resolve an object depends on the level of contrast in the object and its size, reconstruction algorithm, image noise and window settings used to display the image. Spatial resolution is the ability to differentiate small objects with high contrast compared to the background. It characterizes the imaging system's ability to distinguish between two very small objects placed closely together. Spatial resolution measurements are performed with objects which have a high contrast (contrast difference of $12 \%$ or greater) from uniform background. The uniformity describes how uniform the image of a homogenous material appears. The uniformity measurements are important to ensure that cupping and beam hardening artifacts are avoided. CT images are generated by measurement of attenuation of $\mathrm{x}$-rays through the tissue of interest. Attenuation is described by CT-numbers or Hounsfield units (HU). Each pixel in the image corresponds to a specific $\mathrm{HU}$ [2].

Publications by the International Electrotechnical Commission (IEC), the American Association of Physicists in Medicine (AAPM) and the Institute of Physics and Engineering in Medicine (IPEM) form a basis for the monitoring of CT systems. IEC publish recommendations and acceptance values for international use, and provide assistance in performing QA-tests on CT-scanners. IEC recommends the mean CT-number of a central region of interest (ROI) in a uniformity device not to deviate by more than $4 \mathrm{HU}$ from the nominal values specified by the manufacturer of the CT-scanner for the specific material of the test device [3].

The uniformity (the deviation in mean CT-number between central and peripheral regions) must not be greater than $4 \mathrm{HU}$ at acceptance [2] [3]. In addition, the difference in uniformity should not vary by more than $2 \mathrm{HU}$ from baseline values [4]. With respect to low contrast resolution, the detectability should meet the specifications of the manufacturer, but because of difficulty in objectively measuring low contrast resolution IEC does not recommend this method as an acceptance test. IPEM suggests a level of 5HU from the baseline CT-number of water and $10 \mathrm{HU}$ from the baseline number of other materials. The suspension levels are $20 \mathrm{HU}$ and $30 \mathrm{HU}$, respectively. According to IPEM the uniformity should not exceed 10 or $20 \mathrm{HU}$, depending on phantom size [5].

Different types of Image phantoms are used worldwide to evaluate image performance of CT-scanners. The Catphan Phantoms manufactured by the Phantom Laboratory are certainly the most popular. One of those phantoms, the Catphan 700 phantom is a diagnostic imaging tool specially designed for comprehensive evaluation of axial, spiral, multi-slice, cone beam, and volume CT scanners from the point of view of maximum performance [6].

Garayoa and Castro [7] performed a study to evaluate the image quality on a cone beam CT scanner by determining various physical parameters that characterize a system's performance, using Catphan 700 phantom. Eric Naab Manson et al. [8] used a Catphan 700 phantom to assess some image quality tests on a 
128 slice computed tomography scanner. Gulliksrud K, et al. [9] also used a Catphan 700 phantom and other phantoms to carry out some image quality control tests on a Toshiba CT-scanner. Elnour et al. used a Catphan 500/600 phantom to assess image quality parameters for Computed Tomography in Sudan [10].

The assessment of CT scanners image quality performance with Catphan phantoms is very important since those phantoms are also used by the manufacturers to test the image performances of theirs CT scanners after their manufacturing. This enables a comparison with the results obtained by the manufacturers.

\section{Materials and Methods}

To perform the experimental study, 05 CT scanners were analyzed in Yaoundé a city of about three millions of inhabitants. The total number of CT scanners in the city of Yaoundé is 10, however due to regular failures all the CT scanners are not always available. The sample consisted of different manufacturers and models of CT scanners, as shown in Table 1.

A phantom, manufactured by Phantom Laboratory, Catphan model 700, serial number 700158 [9] was used in this study. The Catphan700 is a six modules image phantom. The six modules CTP682, CTP714, CTP515, CTP721, CTP723 and CTP712 are respectively called geometry sensitometry module, 30 line pair resolution module, low contrast module, wave insert module, bead blocks module and uniformity module. The module CTP682 was used for radiation profile and CT number accuracy, the module CTP 714 was used for spatial resolution, the module CTP 515 was used for low contrast resolution and the module CTP 712 was used to test the uniformity.

The CT scanners were evaluated in five quality control tests in order to verify theirs adequacy.

\subsection{Radiation Profile Width}

The geometry sensitometry module CTP682 has a pair of $23^{\circ}$ wire ramps oriented parallel to the $\mathrm{x}$-axis at $0^{\circ}$ rotation. These wire ramps were used to measure slice widths. The radiation profile width aimed to verify that it meets IAEA specifications. Following the related IAEA publication, the tolerance limit

Table 1. Characteristics of CT scanners evaluated.

\begin{tabular}{ccc}
\hline CT scanner $\mathrm{n}^{\circ}$ & Manufacturer/Model \\
\hline 1 & Toshiba/Aquilion 16 \\
2 & Toshiba/Alexion \\
3 & Neusoft/Neuviz 16 \\
4 & Toshiba/Aquilion 16 \\
5 & Toshiba/Aquilion 16 \\
\hline
\end{tabular}


is $1 \mathrm{~mm}$ for slice widths upper than $2 \mathrm{~mm}$.

\subsection{Field Uniformity}

The consistency of the CT-numbers in the image of a homogenous material was measured in the uniformity module (CTP712) in the phantoms. Uniformity of the CT-numbers was measured manually by placing ROIs, one in the middle and four in the periphery of the module (clock positions 12, 3, 6 and 9). The observer used ROIs with a diameter of $10 \%$ of the diameter of the image of the uniformity module, in agreement with IEC 61223-3-5. As stated in the IAEA publication, tolerance limit for uniformity is $\pm 10 \mathrm{HU}$.

\subsection{CT Numbers}

The geometry sensitometry module CTP682 contains inserts with different known densities for measurements of CT-numbers and linearity of CT-numbers for different mass densities. Catphan 700 has inserts made from teflon, acrylic, low density polyethylene (LDPE), air, polymethylpentene (PMP), delrin, bone $20 \%$, bone $50 \%$ and polystyrene. It also has a water container that was filled with distilled water.

The procedure to evaluate the accuracy of the CT number is similar to the test for evaluation of field uniformity. After scanning the uniform section of the phantom, the ROI tool is used to measure the mean CT number for water and other materials. The IAEA publication [1] suggests a tolerance limit of $\pm 5 \mathrm{HU}$ for all CT numbers and the Catphan 700 manual [6] gives tolerance ranges of CT numbers for the different inserts materials.

\subsection{Spatial Resolution (High Contrast Resolution)}

Module 714 contains the 30 line pair/cm gauge for visual evaluation of high resolution ranging from 1 through 30 line pair $/ \mathrm{cm}$. Spatial resolution was measured by counting the numbers of resolved group of $\mathrm{Lp} / \mathrm{cm}$, assessed by three independent investigators.

The scan parameters in this test were as recommended by each manufacturer of the CT scanner. The value of the spatial resolution measured was then compared with the value specified by the manufacturer. as recommended by the IAEA publication [1].

\subsection{Contrast Resolution (Low Contrast Resolution)}

Module CTP515 contains low contrast subslice and supraslice targets with diameters 2 - $15 \mathrm{~mm}$, and contrast levels of $0.3 \%, 0.5 \%$ and $1.0 \%$, used to evaluate the ability to differentiate objects with slightly different densities. Low contrast was measured by counting numbers of visible targets, assessed by three independent investigators.

The scan parameters in this test were as recommended by each manufacturer of the CT scanner. The value of the low contrast resolution measured was then 
compared with the value specified by the manufacturer. as recommended by the IAEA publication [1].

\section{Results}

\subsection{Radiation Profile Width}

The nominal CT slice width in this test was $2 \mathrm{~mm}$, the actual slice width was measured for each CT scanner, and the results are shown on Table 2.

For all the CT scanners, the difference between the measured slice width and the nominal slice width is less than $1 \mathrm{~mm}$. Therefore all the CT-scanners pass this test.

\subsection{Assessment of Spatial Resolution}

The results of this test are shown on Table 3.

It appears that the measured spatial resolution for each of the five CT scanner is far below the manufacturer's spatial resolution. However no CT scanner showed a spatial resolution of less than $8 \mathrm{Lp} / \mathrm{cm}$. This shows that the equipment can still detail objects of small size and high contrast, such as small kidney stones [11].

\subsection{Low Contrast Resolution}

The results of this test are shown on Table 4.

The low contrast resolution for CT scanner 3 was as expected, but the low contrast resolution for the others CT scanners was lower than the manufacturer's low contrast.

Table 2. Radiation profile with a nominal diameter of $2 \mathrm{~mm}$.

\begin{tabular}{cc}
\hline CT-scanner $^{\circ}$ & Measured slice width \\
\hline 1 & $2.10 \mathrm{~mm}$ \\
2 & $2.40 \mathrm{~mm}$ \\
3 & $1.93 \mathrm{~mm}$ \\
4 & $2.50 \mathrm{~mm}$ \\
5 & $1.97 \mathrm{~mm}$ \\
\hline
\end{tabular}

Table 3. Assessment of spatial resolution

\begin{tabular}{ccc}
\hline CT-scanner $\mathrm{n}^{\circ}$ & Measured spatial resolution & Manufacturer's spatial resolution \\
\hline 1 & $10 \mathrm{Lp} / \mathrm{cm}$ & $18 \mathrm{Lp} / \mathrm{cm}$ \\
2 & $9 \mathrm{Lp} / \mathrm{cm}$ & $18 \mathrm{Lp} / \mathrm{cm}$ \\
3 & $9 \mathrm{Lp} / \mathrm{cm}$ & $15 \mathrm{Lp} / \mathrm{cm}$ \\
4 & $13 \mathrm{Lp} / \mathrm{cm}$ & $18 \mathrm{Lp} / \mathrm{cm}$ \\
5 & $12 \mathrm{Lp} / \mathrm{cm}$ & $18 \mathrm{Lp} / \mathrm{cm}$ \\
\hline
\end{tabular}




\subsection{Field Uniformity}

The results of this test are shown on Table 5. The field uniformity measured is less than the acceptable value of $10 \mathrm{HU}$ recommended by the IAEA. Therefore all the CT scanners pass this test.

\subsection{CT Numbers}

The measured CT numbers are shown on Table 6.

Table 4. Assessment of low contrast resolution.

\begin{tabular}{ccc}
\hline CT-scanner ${ }^{\circ}$ & Measured low contrast resolution & Manufacturer's low contrast resolution \\
\hline 1 & $7 \mathrm{~mm} @ 0.3 \%$ & $3 \mathrm{~mm} @ 0.3 \%$ \\
2 & $5 \mathrm{~mm} @ 0.3 \%$ & $3 \mathrm{~mm} @ 0.3 \%$ \\
3 & $4 \mathrm{~mm} @ 0.3 \%$ & $4 \mathrm{~mm} @ 0.3 \%$ \\
4 & $5 \mathrm{~mm} @ 1 \%$ & $3 \mathrm{~mm} @ 0.3 \%$ \\
5 & $4 \mathrm{~mm} @ 0.3 \%$ & $3 \mathrm{~mm} @ 0.3 \%$ \\
\hline
\end{tabular}

Table 5. Assessment of CT field uniformity.

\begin{tabular}{cc}
\hline CT-scanner $^{\circ}$ & Measured field uniformity \\
\hline 1 & $2.4 \mathrm{HU}$ \\
2 & $4.2 \mathrm{HU}$ \\
3 & $1.64 \mathrm{HU}$ \\
4 & $2.73 \mathrm{HU}$ \\
5 & $1.1 \mathrm{HU}$ \\
\hline
\end{tabular}

Table 6. Measured CT numbers in Hounsfield Unit.

\begin{tabular}{|c|c|c|c|c|c|c|c|}
\hline \multirow{3}{*}{ Material } & \multirow{3}{*}{$\mu\left(\mathrm{cm}^{-1}\right)$} & \multicolumn{5}{|c|}{ CT-numbers (HU) } & \multirow{3}{*}{ HU Range } \\
\hline & & \multicolumn{5}{|c|}{ CT-scanner $n^{\circ}$} & \\
\hline & & 1 & 2 & 3 & 4 & 5 & \\
\hline Air & $1.89 \mathrm{E}-04$ & -991.4 & -996.6 & -971.48 & -1026.62 & -1010.4 & $-1046:-986$ \\
\hline Lung & $2.87 \mathrm{E}-02$ & -823.2 & -827.3 & -805.61 & -833.01 & -838.3 & $-925:-810$ \\
\hline PMP & $1.36 \mathrm{E}-01$ & -188.8 & -189.9 & -181.24 & -183.06 & -189.8 & $-220:-172$ \\
\hline LDPE & $1.51 \mathrm{E}-01$ & -100.8 & -107.2 & -92.18 & -93.69 & -100.5 & $-121:-87$ \\
\hline Polystyrène & $1.59 \mathrm{E}-01$ & -41.6 & -55.5 & -35.85 & -26.39 & -40.2 & $-65:-29$ \\
\hline Water & $1.61 \mathrm{E}-01$ & -1.4 & -1.9 & -2.43 & 7.19 & 1.7 & $-7: 7$ \\
\hline Acrylic & $1.84 \mathrm{E}-01$ & 119.5 & 122.5 & 111.16 & 126.98 & 118.6 & $92: 137$ \\
\hline Bone $20 \%$ & $1.78 \mathrm{E}-01$ & 239.1 & 242.7 & 222.63 & 260.24 & 248.2 & $211: 263$ \\
\hline Delrin $^{\oplus}$ & $2.19 \mathrm{E}-01$ & 321.3 & 325.3 & 339.18 & 363.13 & 356.1 & $344: 387$ \\
\hline Bone $50 \%$ & $2.25 \mathrm{E}-01$ & 669.7 & 669.6 & 640.62 & 749.11 & 720.4 & $667: 783$ \\
\hline Teflon & $3.05 \mathrm{E}-01$ & 922.6 & 981.37 & 960.22 & 981.37 & 967.5 & $941: 1060$ \\
\hline
\end{tabular}




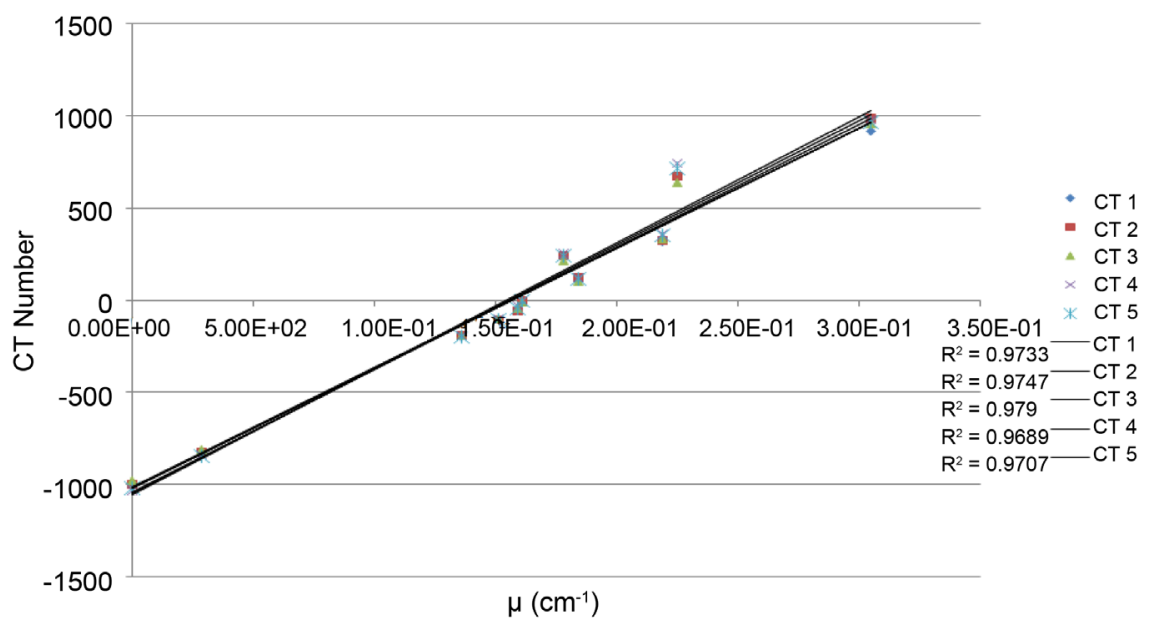

Figure 1. The measured computed tomography numbers of the target materials plotted against linear attenuation coefficients for tube high voltage of $120 \mathrm{kV}$.

The CT numbers for CT-scanner 5 was within the expected range as predicted in Catphan 700 manual. The CT numbers on others CT-scanners were found to be accurate for the majority of materials except for Delrin@, Polystyrene, Teflon, Bone 20\% and Bone 50\%. The CT number of Delrin@ was out of range for CT-scanners 1 and 2. The CT number of Polystyrene was out of range for CT-scanner 4. The CT number of Teflon was out of range for CT-scanner 1. The CT number of Bone $20 \%$ was out of range for CT-scanner 2. The CT number of Bone $50 \%$ was out of range for CT-scanner 3 .

To establish a constancy of contrast scale over the range of CT numbers which is of clinical interest, the CT linearity was verified. This was performed by checking whether the CT numbers measured vary in a linear fashion with their linear attenuation coefficient values. [10] Clinically, CT number relevant linearity was found to be above $\mathrm{R}^{2}=0.968$ close to unity for all the Ct-scanners as observed in Figure 1.

\section{Conclusion}

From the analysis of tests, it was observed that the five CT scanners showed a high rate of approval for three of the five tests performed. The measured spatial resolution for each of the five CT scanners was far below the spatial resolution specified by the manufacturer. The low contrast resolution of four of the five CT scanners was found low when compared to the low contrast resolution specified by the manufacturer. However the measured spatial resolution was above the minimal value required for the performance of thoracic computed tomography [11].

\section{References}

[1] International Atomic Energy Agency, Quality Assurance Program for Computed Tomography; Diagnostic and Therapy Applications, IAEA Human Health Series $\mathrm{N}^{\circ}$ 11, IAEA, Vienna (2012). 
[2] Birnbaum, B.A., Hindman, N., Lee, J. and Babb, J.S. (2007) Multi-Detector Row CT Attenuation Measurements: Assessment of Intra- and Interscanner Variability with an Anthropomorphic Body CT Phantom. Radiology, 242, 109-119. https://doi.org/10.1148/radiol.2421052066

[3] IEC 61223-3-5. International Standard IEC 61223-3-5. Evaluation and Routine Testing in Medical Imaging Departments-Part 3 - 5: Acceptance Tests-Imaging Performance of Computed Tomography X-Ray Equipment; 2013.

[4] IEC 61223-2-6. International Standard IEC 61223-2-6. Evaluation and Routine Testing in Medical Imaging Departments-Part 2-6: Acceptance Tests-Imaging Performance of Computed Tomography X-Ray Equipment; 2006.

[5] IPEM (2005) Report 91.

[6] The Phantom Laboratory, United States: Catphan ${ }^{\circledR} 700$ Manual. @2013. http://www.phantomlab.com/catphan-phantoms/

[7] Garayoa, J. and Castro, P. (2013) A Study on Image Quality Provided by a Kilovoltage Cone-Beam Computed Tomography. Journal of Applied Clinical Medical Physics, 14, 3888. https://doi.org/10.1120/jacmp.v14i1.3888

[8] Manson, E.N., et al. (2016) Assessment of Some Image Quality Tests on a 128 Slice Computed Tomography Scanner Using a Catphan700 Phantom. Journal of Medical Physics, 41, 153-156. https://doi.org/10.4103/0971-6203.181637

[9] Gulliksrud, K., Stokkea, C. and Martinsen, A.C.T. (2014) How to Measure CT Image Quality: Variations in CT-Numbers, Uniformity and Low Contrast Resolution for a CT Quality Assurance Phantom. Physica Medica.

[10] Elnour, H., Hassan, H.A., Mustafa, A., Osman, H., Alamri, S. and Yasen, A. (2017) Assessment of Image Quality Parameters for Computed Tomography in Sudan. Open Journal of Radiology, 7, 75-84. https://doi.org/10.4236/ojrad.2017.71009

[11] American College of Radiology, ACR-SCBT-MR-SPR Practice Parameter for the Performance of Computed Tomography (CT) of Computed Tomography (CT) of the Pelvis. 2016.

https://www.acr.org/-/media/ACR/Files/Practice-Parameters/ct-abd-pel.pdf 